\title{
The Start of Something New
}

This first issue of a new international journal is the culmination of efforts by many people. The need for such a communication vehicle was brought to our attention by the Executive Council of the International Round Table for the Advancement of Counselling.

Through consultation and through 'Round Table' conferences, IRTAC has played a significant role in the development and shaping of counselling services in many areas of the world. It has been primarily because of the success of these endeavours, that the Executive Council of IRTAC sought ways to broaden the communication base of the Association, to keep counsellors aware of international developments on a continuous basis, not only at conferences, and through conference proceedings.

Thus, the present editors were appointed, to find out the feasibility of founding an international journal, with a major aim of improving communication to bring counsellors from different cultures closer to developments in all parts of the world. It is through this journal, that the editors hope to accomplish this goal.

The journal will continue to include major papers from 'Round Table' conferences, as is the case in this issue. In this way our readers will be kept informed of the latest thinking of some of the recognized 'giants' of our profession. The journal will also include informed comment on deliberations of working groups to help provide 'reaction stimulus' to major ideas and themes debated within the context of Round Table seminars.

The major focus of the journal will be to present ideas and descriptions of counselling problems and practices throughout the world. We welcome original articles from practitioners, educators and students, with the sincere belief that counsellors throughout the world will be enriched and enlightened through the sharing of both unique and common problems; familiar and unfamiliar solutions.

It is anticipated that the editors will maintain a close relationship with IRTAC, but the journal will be independent from that association. The names of our Editorial Board members appear elsewhere in this issue, but responsibility for journal content and format rests with the editors.

This first issue publishes some of the most important contributions to the 
last Round Table conferences, held in Würzburg and Cambridge. As those contributions have not explicitly been written with the purpose of publication, their form is not always representative of what the Editors have in mind for the future. We expect to publish abstracts of most articles in a main second language in future issues.

We invite your comments, suggestions and criticisms. Most important, we welcome your contributions to future issues. The editors are committed to presenting international ideas; this can only be done if readers from all parts of the world join with us to enrich the profession through shared ideas.

Our thanks to the valued contributors to our first issue. And special thanks to Hans Hoxter, who makes difficult tasks possible, and to whom we dedicate this first issue of the International Journal for the Advancement of Counselling.

The Editors 